\title{
IPTV: Technology, Practice, and Service
}

\author{
Hsiang-Fu Yu, ${ }^{1}$ Jen-Wen Ding, ${ }^{2}$ Pin-Han Ho, ${ }^{3}$ and János Tapolcai ${ }^{4}$ \\ ${ }^{1}$ Department of Computer Science, National Taipei University of Education, Taipei 106, Taiwan \\ ${ }^{2}$ Department of Information Management, National Kaohsiung University of Applied Sciences, Kaohsiung 807, Taiwan \\ ${ }^{3}$ Department of Electrical and Computer Engineering, University of Waterloo, Waterloo, ON, Canada N2L 3G1 \\ ${ }^{4}$ Department of Telecommunication and Media Informatics, Budapest University of Technology and Economics, \\ Budapest 1521, Hungary
}

Correspondence should be addressed to Hsiang-Fu Yu, yu@tea.ntue.edu.tw

Received 10 July 2012; Accepted 10 July 2012

Copyright (c) 2012 Hsiang-Fu Yu et al. This is an open access article distributed under the Creative Commons Attribution License, which permits unrestricted use, distribution, and reproduction in any medium, provided the original work is properly cited.

With the advances in video compression and broadband access technologies, the Internet Protocol Television (IPTV) becomes a popular technology for the delivery of multimedia services directly to the end users. A number of operators and vendors are currently working on IPTV standardization efforts (e.g., ATIS/IIF, ITU-T FG IPTV) to support IPTV as a secure, reliable, and managed service. IPTV also provides bundled service offerings that encompass Internet access, telephony (VoIP), multimedia services, and mobile services. Being regarded as a great business opportunity for content providers, service providers, and equipment manufacturers, there are still many issues in the standardization, design, development, and deployment of commercially viable IPTV services.

The objective of this special issue is to provide a forum for sharing knowledge and recent advances in IPTV services. A total of 14 high-quality submissions were received for this special issue. However, we are only able to accept 8 of them due to the page limit.

In the paper entitled "A secure and stable multicast overlay network with load balancing for scalable IPTV services," T.-T. Wei et al. propose a secure application-layer multicast overlay network for IPTV, called SIPTVMON. SIPTVMON can secure all the IPTV media delivery paths against eavesdroppers via elliptic-curve Diffie-Hellman (ECDH) key exchange on SIP signaling and AES encryption. The performance analysis shows that SIPTVMON outperforms in quality of privacy protection, stability from user churn, and good perceptual quality for scalable IPTV services over Internet.

In the paper entitled "Video classification and adaptive QoP/QoS control for multiresolution video applications on IPTV," S.-F. Huang presents a quality control mechanism in multiresolution video coding structures over WIMAX networks. This work also investigates the relationship between QoP and QoS in end-to-end connections.

In the paper entitled "Adjustable two-tier cache for IPTV based on segmented streaming," K.-C. Liang and H.-F. Yu propose a segment-based two-tier caching approach, which divides each video into multiple segments to be cached. This approach also partitions the cache space into two layers, where the first layer mainly caches to-be-played segments and the second layer saves possibly played segments. Comprehensive simulation results show that the approach can yield higher hit ratio than previous work under various environmental parameters.

In the paper entitled "A seamless broadcasting scheme with live video support," Z.-Y. Yang et al. present a scalable binomial broadcasting scheme to transfer live videos using constant bandwidth by increasing clients' waiting time. When the scheme finds that the length of a video exceeds the default, it doubles the length of to-be-played segments, and then its required bandwidth is constant.

In the paper entitled "An efficient periodic broadcasting with small latency and buffer demand for near video on demand," Y.-N. Chen and L.-M. Tseng improve the fixeddelay pagoda broadcasting (FDPB) scheme to save client buffering space as well as waiting time. In comparison with the staircase broadcasting (SB), the reverse fast broadcasting (RFB), and the hybrid broadcasting ( $\mathrm{HyB})$ schemes, the improved FDPB scheme can yield the smallest waiting time under the same buffer requirements.

In the paper entitled "QoS supported IPTV service architecture over hybrid-tree based explicit routed multicast network," C.-C. Wen and C.-S. Wu propose a cooperative 
scheme of hybrid-tree based on explicit routed multicast, called as HT-ERM to combine the advantages of shared tree and source tree for QoS supported IPTV service. The simulation results show that the HT-ERM scheme outperforms other multicast QoS-based delivery schemes in terms of channel switching delay, resource utilization, and blocking ratio for IPTV services.

In the paper entitled "Background traffic-based retransmission algorithm for multimedia streaming transfer over concurrent multipaths," Y. Cao et al. firstly investigate the effect of background traffic on the performance of concurrent multipath transfer SCTP (CMT-SCTP). Motivated by the localness nature of background flow, this work proposes an improved retransmission algorithm named RTX_CSI to yield higher average throughput and better users' experience of quality for multimedia streaming services.

In the paper entitled "Secure and reliable IPTV multimedia transmission using forward error correction," C.-H. Shih et al. propose a novel FEC scheme to ensure the secure and reliable transmission for IPTV multimedia content and services. The scheme utilizes the characteristics of FEC including the FEC-encoded redundancies and the limitation of error correction capacity to protect the multimedia packets against the malicious attacks and the data transmission errors/losses.

As we conclude this overview, we would like to express our sincere appreciation to all the reviewers for their timely and insightful comments on the submitted papers, which made this special issue possible.

Hsiang-Fu $\mathrm{Yu}$ Jen-Wen Ding

Pin-Han Ho

János Tapolcai 

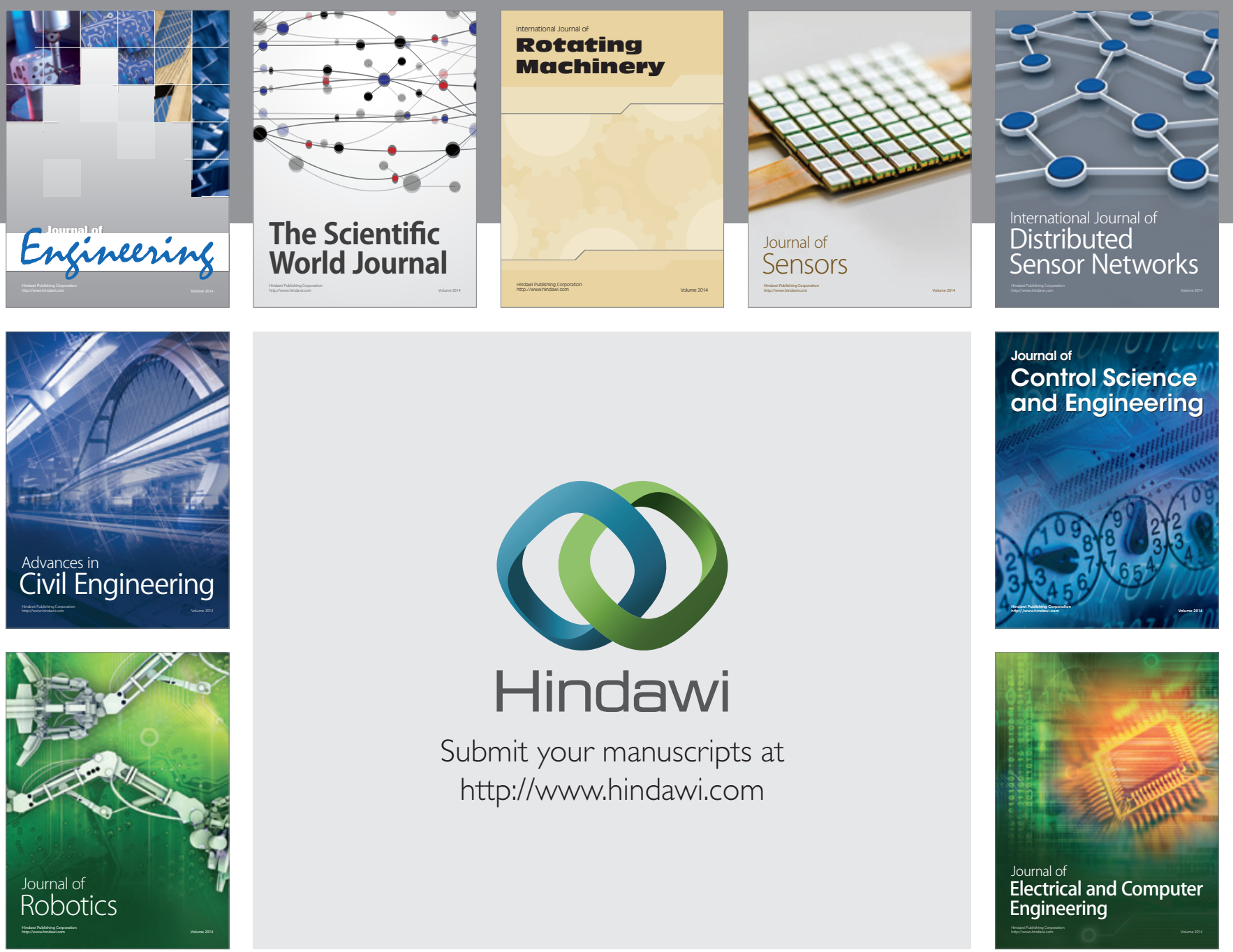

Submit your manuscripts at

http://www.hindawi.com
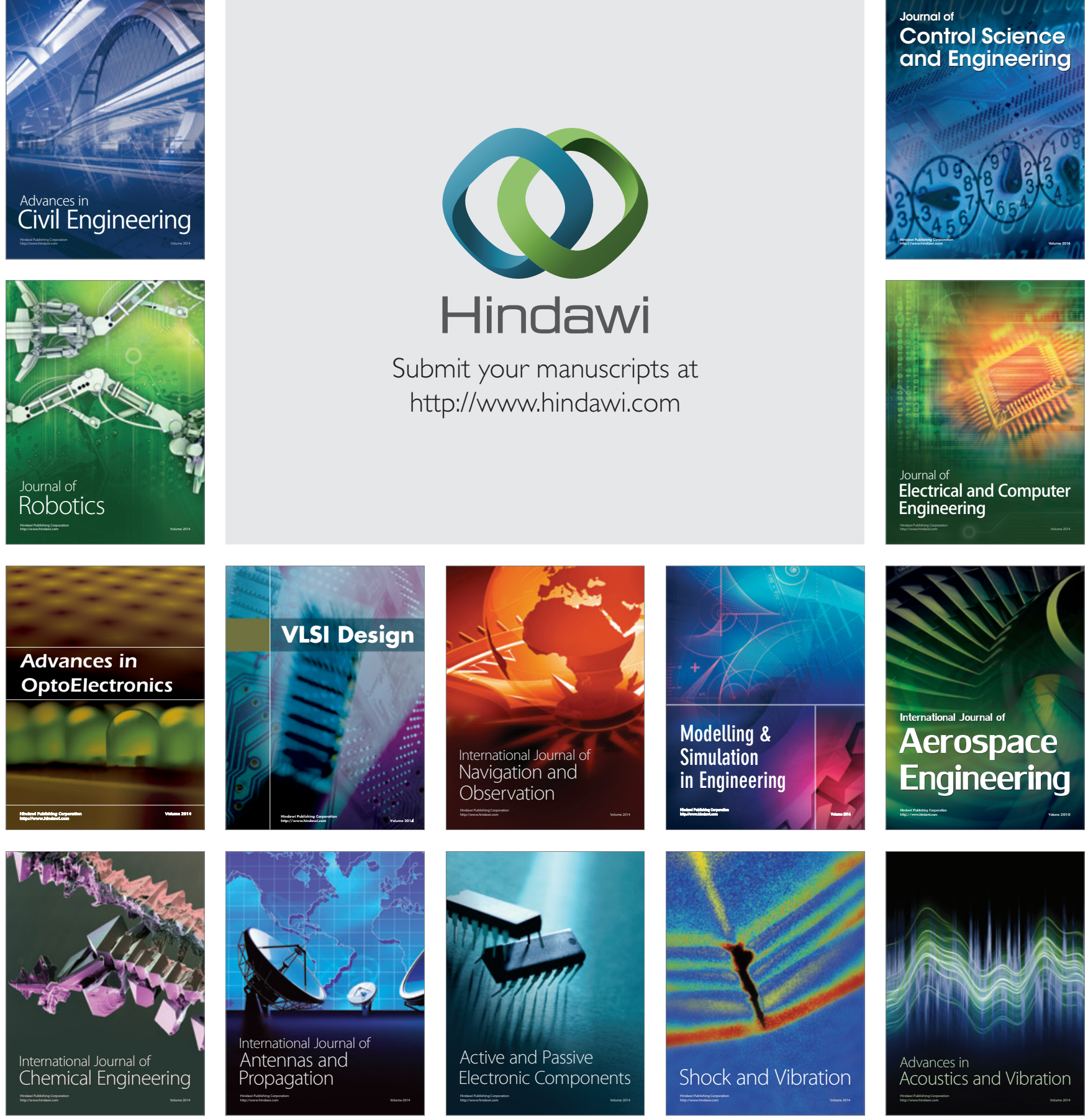